\title{
Tuberculosis Contact Screening and Isoniazid Preventive Therapy Among Children Under 5 in the Mbeya and Songwe Regions, Tanzania
}

\author{
Yesaya K Mwasubila, ${ }^{a, b}$ Issa Sabi, ${ }^{c}$ Rogatus Kabyemera, ${ }^{d, e}$ Nyanda E Ntinginya, ${ }^{c}$ Reginald Sauve, ${ }^{b, f}$ \\ Benson Kidenya ${ }^{g}$ \\ a Department of Health, Songea District Council, Ruvuma, Tanzania; ${ }^{b}$ School of Public Health, Catholic University of Health and Allied Sciences, Mwanza, Tanzania; \\ 'Mbeya Medical Research Centre, National Institute for Medical Research, Mbeya, Tanzania; ' Department of Paediatrics and Child Health, Bugando Medical Centre, \\ Mwanza, Tanzania; ${ }^{e}$ Department of Paediatrics and Child Health, Catholic University of Health and Allied Sciences, Mwanza, Tanzania; ${ }^{f}$ Faculty of Medicine, \\ University of Calgary, Calgary, Alberta, Canada; ${ }^{9}$ Department of Biochemistry, Catholic University of Health and Allied Sciences, Mwanza, Tanzania \\ Correspondence to Issa Sabi (isabi@nimr-mmrc.org)
}

\begin{abstract}
Introduction: The World Health Organization (WHO) recommends contact screening and initiation of isoniazid preventive therapy (IPT) for children under 5 years of age exposed to a sputum smear-positive (SS+) tuberculosis (TB) source case. We conducted this study in order to assess implementation of these recommendations in southwestern Tanzania.

Methods: We conducted this cross-sectional study from June to August 2015 in 12 selected health facilities in the Mbeya and Songwe regions of Tanzania. Adult SS+ pulmonary TB patients living in the same household as children under 5 were enrolled. Structured questionnaires were used to obtain sociodemographic information and details about screening and intervention activity related to contact children under 5. Data were analysed using Stata version 11.0.

Results: We enrolled 257 index cases, who collectively had 433 contact children under 5 . The median age of the index cases was 34 years (interquartile range 28 to 41 ) and 52.9\% were male. Out of 433 contacts, 31 (7.2\%) were screened for TB, of whom $7(22.6 \%)$ were treated for presumptive TB. Among those screened, 24 were not diagnosed with TB, of whom only $8(33.3 \%)$ received IPT.

Conclusion: Low uptake of TB contact screening and IPT administration among eligible children under 5 was observed in this study. Health-care workers should be sensitized to screening of household contacts of adults with SS+ TB and initiate IPT in those who are eligible.
\end{abstract}

\section{INTRODUCTION}

C hildhood tuberculosis (TB) contributes a significant proportion of the global TB burden. In 2015, 10\% of the 10.4 million incident TB cases worldwide occurred in children under 15 years of age. ${ }^{1}$ Children are usually infected via household contact with an adult who has sputum smear-positive (SS+) pulmonary TB (PTB). ${ }^{2}$

In line with recommendations set by the World Health Organization (WHO) and governed by national TB programmes, strategies for preventing childhood TB include contact tracing and isoniazid preventive therapy (IPT) for children under 5 in contact with TB source cases. ${ }^{3,4}$ IPT has been shown to reduce the risk of progression from latent TB infection to active TB disease by as much as 59\% among children aged 15 years or younger. $^{5-7}$ Despite the known benefits of contact screening and IPT, these strategies remain widely underutilized, even in countries severely affected by TB. ${ }^{8-12}$ For example, a study in Malawi found that only $9 \%$ of contacts were screened for TB and only $6 \%$ were initiated on IPT. ${ }^{13}$

Tanzania is among the world's 22 high TB-burden countries and childhood TB is estimated to contribute about $10 \%$ of the national TB burden. ${ }^{1}$ In keeping with WHO recommendations, Tanzania's National Tuberculosis and Leprosy Control Programme (NTLP) recommends contact investigation and IPT provision for all TB contacts under 5, after excluding active TB disease. ${ }^{4}$ TB treatment services, including IPT initiation and follow-up, are provided for free by the NTLP and supervised by district $\mathrm{TB}$ and leprosy coordinators 
(DTLCs), under the guidance of regional TB and leprosy coordinators (RTLCs). ${ }^{4}$ Despite these recommendations and deployments, uptake of contact tracing and IPT initiation has not been well documented in most facilities, which has made it difficult to evaluate performance and adherence to guidelines. We conducted this study in 2 regions of Tanzania to assess uptake of contact screening and IPT administration, in 2 regions of Tanzania, for children under 5 who are contacts of SS + TB patients.

\section{METHODS}

\section{Study Design and Setting}

Data for this cross-sectional study were collected from June to August 2015 in the Mbeya and Songwe regions located in the Southern Highlands Zone in southwestern Tanzania. These regions have TB notification rates ranging from 3,700 to 4,000 cases per year, with childhood TB contributing about $10 \%$ of the total TB burden. At the time of data collection, the 2 regions had 448 health facilities, which included 18 hospitals, 36 health centres, and 394 dispensaries. We randomly selected 10 hospitals and 2 health centres among sites with TB diagnostic and treatment capabilities.

\section{Participants}

We enrolled consenting adult SS + PTB patients who lived in the same household with children under 5. Parents or caregivers who had other forms of TB were excluded. A household contact was defined as a child who shared the same house with an index TB case for at least 3 months prior to diagnosis.

\section{Sample Size and Data Collection}

The minimum sample size was estimated using the following formula: $n=Z^{2} P(1-P) / d^{2}$, where, $Z$ is the level of confidence that the chosen sample was not representative of the population (using the value 1.96 for $95 \%$ confidence interval [CI]), $\mathrm{P}$ represents the proportion of the sample that is assumed to practice contact screening $(50 \%$ or 0.5$)$, and $d$ is margin of error that the probability that the desired sample size was not representative of the study population at $95 \% \mathrm{CI}$, expressed as a decimal (0.05). We estimated the minimum sample size to be 384 contact children under 5 , but included 433 children in the study to minimize errors. Data were obtained through interviews with adult index patients whose clinic TB treatment cards revealed positive TB results from sputum. Participants confirmed the presence of contact children under 5 before consenting to and proceeding with questionnaire-guided face-to-face interviews during routine TB clinic visits. Interviews captured sociodemographic information and details of contact TB screening, prevention with IPT, and treatment.

\section{Data Analysis}

Completed questionnaires were entered into Microsoft Excel and then exported into Stata version 11.0 (Stata Corp, College Station, TX, USA) for analysis. Categorical variables were summarized as proportions and continuous variables as medians with interquartile range (IQR).

\section{Ethical Consideration}

Ethical approval was obtained from the joint Catholic University of Health and Allied Sciences and Bugando Medical Centre (CUHAS/BMC) Ethics and Review Committee. The respective district administrative authorities granted permission to carry out the study. All patients provided written informed consent before participating.

\section{RESULTS}

\section{Characteristics of Tuberculosis Index Cases}

We enrolled 257 index TB cases, who accounted for 433 contact children under 5 . The median age of the index cases was 34 years (IQR 28 to 41 ) and $136(52.9 \%)$ were male. All index cases had at least 1 contact living in the same household and $150(58 \%)$ were biological parents of the contacts (Table 1).

\section{Household Contacts and Isoniazid Preventive Therapy} Out of 433 contacts, only 31 (7.2\%) were reported to have been screened for TB. Among the 31 who were screened, $7(22.6 \%)$ were found to have presumptive TB disease and were treated with anti-TB therapy. Eight of the 24 remaining contacts $(33.3 \%)$ started IPT, while the other 16 eligible contacts did not receive any intervention (Figure 1).

\section{DISCUSSION}

This study investigated the extent of TB contact investigation as an entry point to IPT initiation among children exposed to adult SS + PTB patients in southwestern Tanzania. The main purposes of contact investigation were to identify contacts with presumptive TB disease who require anti-TB therapy and to provide a gateway to IPT for those without presumptive TB but who are susceptible to developing disease following recent infection. ${ }^{7}$

Despite the availability of both WHO and national guidelines, which clearly recommend clinical evaluation of household contacts of SS + PTB adult index cases, only $31(7.2 \%)$ contact children under 5 of SS+ TB source cases were screened for TB. Our findings are similar to screening rates of $9.0 \%$ and $5.5 \%$ among TB contact children under 5 in Malawi and Vietnam, respectively. ${ }^{13,14}$ The low TB screening rate in our study may have left many children without appropriate interventions, putting them at risk of developing active TB disease. 
TABLE 1. Characteristics of Tuberculosis Index Cases in the Mbeya and Songwe Regions, Tanzania

\begin{tabular}{|c|c|c|}
\hline Characteristic & $\begin{array}{l}\text { Frequency }(\mathbf{n}) \\
\text { or median }\end{array}$ & $\%$ or IQR \\
\hline Age, median & 34 & $28-41$ \\
\hline \multicolumn{3}{|l|}{ Sex } \\
\hline Male & 136 & 52.9 \\
\hline \multicolumn{3}{|l|}{ Occupation } \\
\hline Peasant/subsistence farmer & 106 & 41.3 \\
\hline Pet trader & 84 & 32.7 \\
\hline Housewife & 18 & 7.0 \\
\hline Others & 49 & 19.1 \\
\hline \multicolumn{3}{|l|}{ Education } \\
\hline No formal education & 23 & 9.0 \\
\hline Primary education & 190 & 73.9 \\
\hline Secondary education & 36 & 14.0 \\
\hline College education & 8 & 3.1 \\
\hline \multicolumn{3}{|l|}{$\begin{array}{l}\text { Walking time to health facility } \\
\text { on foot (minutes) }\end{array}$} \\
\hline 0 to 30 & 60 & 23.4 \\
\hline 31 to 60 & 66 & 25.7 \\
\hline 61 to 90 & 52 & 20.2 \\
\hline$>90$ & 79 & 30.7 \\
\hline \multicolumn{3}{|c|}{ Number of contacts per household } \\
\hline 1 & 125 & 48.7 \\
\hline 2 & 98 & 38.1 \\
\hline 3 & 25 & 9.7 \\
\hline 4 & 9 & 3.4 \\
\hline \multicolumn{3}{|l|}{ Relation to contact children } \\
\hline Mother or father & 150 & 58.4 \\
\hline Guardian/caregiver & 107 & 41.6 \\
\hline
\end{tabular}

Abbreviations: $I Q R$, interquartile range.

One-third of the IPT-eligible children - those who were screened and found not to have presumptive TB - were started on therapy. This is lower than proportions reported from studies conducted in Ethiopia $(64.3 \%)^{15}$ and South Africa $(68.0 \%),{ }^{16}$ but higher than what has been observed
FIGURE 1. Contact Screening and Isoniazid Preventive Therapy Initiation among Tuberculosis Contact Children Under 5 in the Mbeya and Songwe Regions, Tanzania

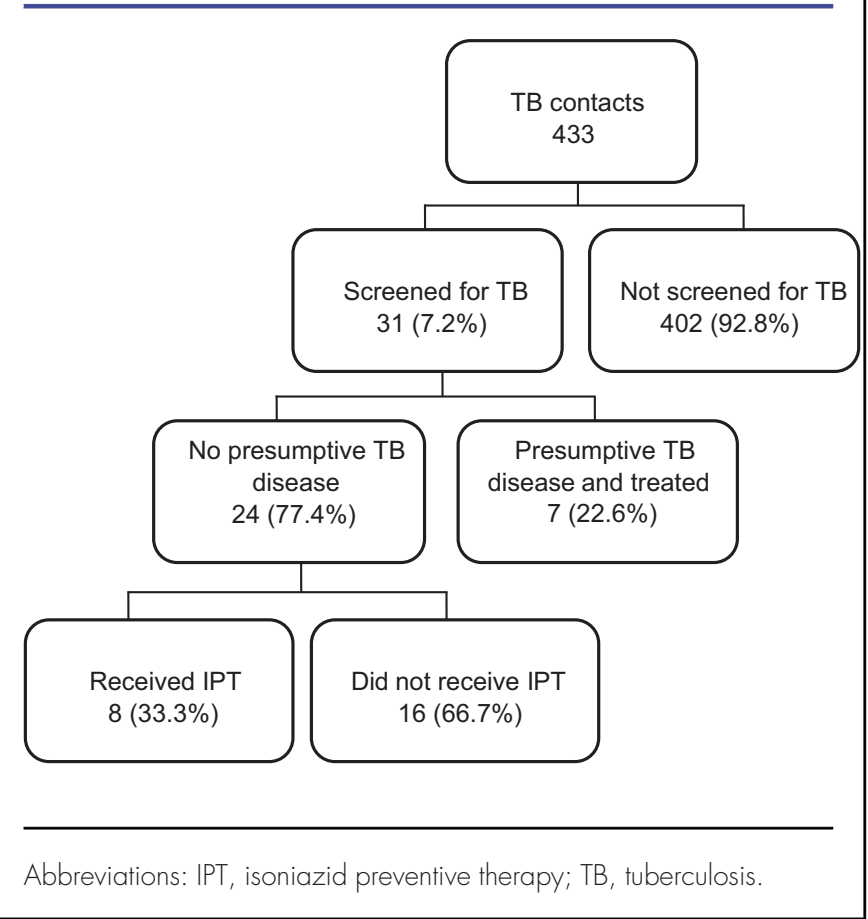

in Malawi $(6.0 \%) .{ }^{13}$ Possible reasons for low IPT uptake in the study area included the limited availability of documentation tools during the study period, which negatively affected the national TB programme's ability to monitor IPT implementation. Contact screening and IPT provision have substantially improved in countries that have introduced IPT registers. For example, in South Africa, the introduction of official documentation forms increased the number of contacts screened per TB patient as well as the proportion of children initiated on IPT. ${ }^{11}$ In India, the introduction of an IPT register increased IPT provision from $19.0 \%$ to $61.0 \%{ }^{17}$ As with the low uptake of contact screening, failure to provide IPT to high proportions - two-thirds in our study - of eligible children may contribute to TB-related morbidity and mortality. In South Africa's Western Cape, for example, a study found that $81.4 \%$ of missed opportunities for IPT in at-risk children under 3 years of age who later presented with confirmed TB $-25.0 \%$ had disseminated TB and $5.1 \%$ died. ${ }^{18}$

\section{Limitations}

This study relied solely on patient recall regarding information about contact tracing, TB screening, and IPT administration. Review of IPT provision data tools may have provided a cross-checking mechanism, but unfortunately, these tools were not available in the study sites during the study period. 
However, this study has provided important evidence to the Tanzanian NTLP on the low uptake of contact investigation and low IPT provision to eligible TB contact children under 5 .

\section{CONCLUSION}

The uptake of contact screening and IPT administration among children under 5 observed in this study was low. We advise the NTLP to fully implement the IPT programme and sensitize health-care workers on the importance of contact screening and IPT for those who are eligible. Further research is needed to inform the national TB programme on factors associated with low uptake of contact investigation by health-care workers and the feasibility of implementation of IPT recording tools under the programme setting.

Acknowledgements: The authors thank the patients who volunteered to take part in the study. We acknowledge the support received from the RTLC and DTLC offices and the health-care workers in the TB treatment clinics in the Mbeya and Songwe regions. This research was supported by the Tanzanian Ministry of Health and Social Welfare through an unconditional grant to YKM.

\section{REFERENCES}

1. World Health Organization (WHO). Global Tuberculosis Report 2016. Geneva: WHO; 2016. http://apps.who.int/medicinedocs/en/d/Js23098en/. Accessed 19 March 2018.

2. Claessens NJM, Gausi FF, Meiinen S, Weismuller MM, Salaniponi FM, Harries AD. High frequency of tuberculosis in households of index TB patients. Int $J$ Tuberc Lung Dis. 2002;6(3):266-269. Medline

3. World Health Organization (WHO). Recommendations for Investigating Contacts of Persons with Infectious Tuberculosis in Low-and Middle-Income Countries. Geneva: WHO; 2012. http://www.who.int/tb/publications/2012/ contact_investigation2012/en/. Accessed 19 March 2018.

4. Tanzania Ministry of Health and Social Welfare, National Tuberculosis and Leprosy Control Programme (NTLP). Tanzania National Guidelines for the Management of Tuberculosis in Children. Dar es Salaam, Tanzania: NTLP; 2013. http://ihi.eprints. org $/ 3363 / 1 /$ national_guidelines_for_the_management_of tb_in_children \%281\%29.pdf. Accessed 19 March 2018.

5. Smieja M, Marchetti CA, Cook DJ, Smaill FM. Isoniazid for preventing tuberculosis in non-HIV infected persons. Cochrane Database Syst Rev. 2000;(2):CD001363. Medline

6. Ayieko J, Abuogi L, Simchowitz B, Bukusi EA, Smith AH, Reingold A. Efficacy of isoniazid prophylactic therapy in prevention of tuberculosis in children: a meta-analysis. BMC Infect Dis. 2014;14(1):91. CrossRef. Medline

7. World Health Organization (WHO). Guidance for National Tuberculosis Programmes on the Management of Tuberculosis in Children. 2nd ed. Geneva: WHO; 2014.
8. Weismuller MM, Graham SM, Claessens NJM, Meijnen S, Salaniponi FM, Harries AD. Diagnosis of childhood tuberculosis in Malawi: an audit of hospital practice. Int $J$ Tuberc Lung Dis. 2002;6(5):432-438. Medline

9. Pothukuchi $M$, Nagaraja SB, Kelamane $S$, et al. Tuberculosis contact screening and isoniazid preventive therapy in a South Indian district: operational issues for programmatic consideration. PLoS One. 2011;6(7):e22500. CrossRef. Medline

10. Donald PR, Maher D, Qazi S. A research agenda to promote the management of childhood tuberculosis within national tuberculosis programmes. Int J Tuberc Lung Dis. 2007;1 1(4):370-380. Medline

11. van Soelen N, du Preez K, van Wyk SS, et al. Does an isoniazid prophylaxis register improve tuberculosis contact management in South African children? PLoS One. 2013;8(12):e80803. CrossRef. Medline

12. Rutherford ME, Ruslami R, Anselmo M, et al. Management of children exposed to Mycobacterium tuberculosis: a public health evaluation in West Java, Indonesia. Bull World Health Organ. 2013;91(12):932-941A. CrossRef. Medline

13. Claessens NJM, Gausi FF, Meijnen S, Weismuller MM, Salaniponi FM, Harries AD. Screening childhood contacts of patients with smear-positive pulmonary tuberculosis in Malawi. Int J Tuberc Lung Dis. 2002;6(4):362-364. Medline

14. Thanh THT, Ngoc SD, Viet NN, et al. A household survey on screening practices of household contacts of smear positive tuberculosis patients in Vietnam. BMC Public Health. 2014;14(1):713. CrossRef. Medline

15. Tadesse Y, Gebre N, Daba S, et al. Uptake of isoniazid preventive therapy among under-five children: TB contact investigation as an entry point. PLoS One. 2016; 11(5):e0155525. CrossRef. Medline

16. Schaaf HS, Marais BJ, Whitelaw A, et al. Culture-confirmed childhood tuberculosis in Cape Town, South Africa: a review of 596 cases. BMC Infect Dis. 2007;7(1):140. CrossRef. Medline

17. Rekha B, Jagarajamma K, Chandrasekaran V, Wares F, Sivanandham R, Swaminathan S. Improving screening and chemoprophylaxis among child contacts in India's RNTCP: a pilot study. Int J Tuberc Lung Dis. 2013;17(2):163-168. CrossRef. Medline

18. Du Preez K, Hesseling AC, Mandalakas AM, Marais BJ, Schaaf HS. Opportunities for chemoprophylaxis in children with culture-confirmed tuberculosis. Ann Trop Paediatr. 2011;31(4):301-310. CrossRef. Medline

\section{Peer Reviewed}

Competing Interests: None declared.

Received: 11 Mar 2017; Accepted: 15 Mar 2018

Cite this article as: Mwasubila YK, Sabi I, Kabyemera R, Ntinginya N, Sauve R, Kidenya B. Tuberculosis Contact Screening and Isoniazid Preventive Therapy Among Children Under 5 in the Mbeya and Songwe Regions, Tanzania. East African Health Res J. 2018;2(1):29-32. https://doi.org/10.24248/EAHRJ-D-17-00186

(c) Mwasubila et al. This is an open-access article distributed under the terms of the Creative Commons Attribution License, which permits unrestricted use, distribution, and reproduction in any medium, provided the original author and source are properly cited. To view a copy of the license, visit http://creativecommons.org/licenses/by/ 4.0/. When linking to this article, please use the following permanent link: https:// doi.org/10.24248/EAHRJ-D-17-00186 